Provided for non-commercial research and education use. Not for reproduction, distribution or commercial use.

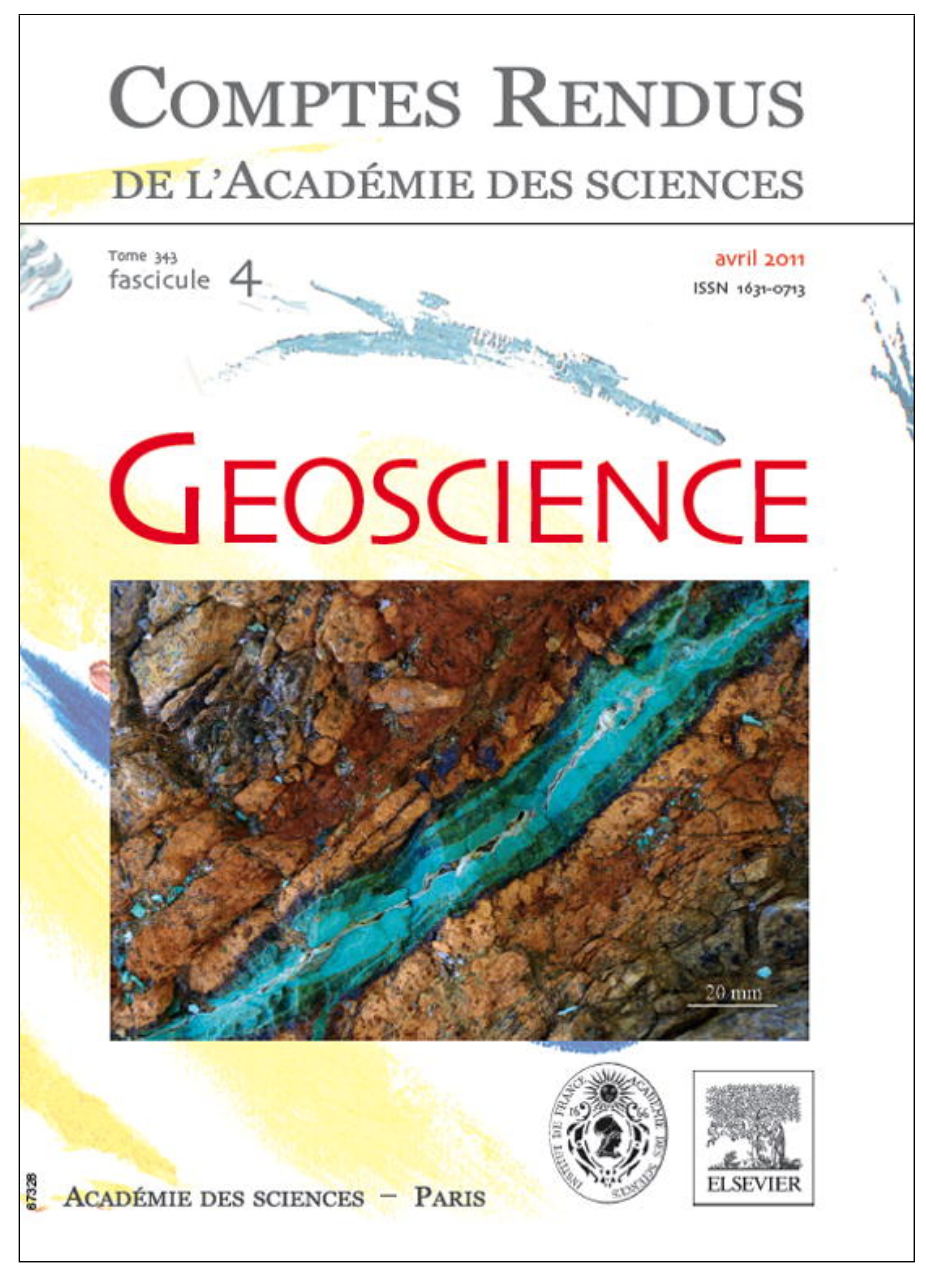

This article appeared in a journal published by Elsevier. The attached copy is furnished to the author for internal non-commercial research and education use, including for instruction at the authors institution and sharing with colleagues.

Other uses, including reproduction and distribution, or selling or licensing copies, or posting to personal, institutional or third party websites are prohibited.

In most cases authors are permitted to post their version of the article (e.g. in Word or Tex form) to their personal website or institutional repository. Authors requiring further information regarding Elsevier's archiving and manuscript policies are encouraged to visit:

http://www.elsevier.com/copyright 
Statigraphie, sédimentologie

\title{
Activités volcaniques sous-marines à la limite Jurassique-Crétacé dans le Rif externe (Maroc). Âge et relation avec la sédimentation et la paléogéographie du sillon rifain externe
}

\section{Submarine volcanism activities at the Jurassic-Cretaceous boundary in the external Rif chain (Morocco). Age and relationship with sedimentation and the palaeogeography of the external riffian trough}

\author{
Mohamed Benzaggagh \\ Université Moulay Ismail, faculté des sciences, département de géologie, BP 11201, Beni M'Hamed, Meknès, Maroc
}

\section{N F O A R T I C L E}

\section{Historique de l'article :}

Reçu le 20 mai 2010

Accepté après révision le 21 décembre 2010

Disponible sur internet le 2 avril 2011

Presenté par Jean Aubouin

\section{Mots clés :}

Stratigraphie

Volcanisme sous-marin

Niveaux volcanoclastiques

Jurassique supérieur

Crétacé inférieur

Rif externe

Maroc

\section{Keywords:}

Stratigraphy

Submarine volcanism

Volcaniclastic levels

Upper Jurassic

Lower Cretaceous

External Rif

Morocco

\begin{abstract}
R É S U M É
À la suite des travaux de stratigraphie menés sur les séries de la limite Jurassique-Crétacé du Rif externe (Mésorif et nappe de Bou Haddoud), de nombreux indices d'activités volcaniques sous-marines ont été découverts dans des niveaux allant du sommet de l'Oxfordien au Berriasien supérieur. Ces indices présentent des aspects variés : complexes volcanoclastiques emballés dans des marnes du Berriasien supérieur ; coulées de laves et lithoclastes de basalte contenus dans des bancs calcaréo-bréchiques du Tithonien inférieur ou dans des brèches à matrice marneuse du sommet de l'Oxfordien à Berriasien inférieur. Ces activités volcaniques sous-marines ont eu lieu dans des conditions de type plate-forme carbonatée peu profonde, pendant le KimméridgienTithonien inférieur ou dans des conditions de type bassin à partir du Tithonien supérieur. Elles ont entraîné, dans plusieurs secteurs, des bréchifications intenses des séries carbonatées du sommet du Jurassique et le démantèlement presque général des alternances marno-calcaires du Tithonien supérieur et partiellement des marnes du Berriasien inférieur.
\end{abstract}

(c) 2011 Académie des sciences. Publié par Elsevier Masson SAS. Tous droits réservés.

\section{A B S T R A C T}

Following the recent stratigraphic works carried out on the Jurassic-Cretaceous boundary in the external Rif chain (Mesorif area and Bou Haddoud nappe), numerous submarine volcanism traces have been discovered in Upper Oxfordian to Upper Berriasian deposits. These traces display various aspects: volcaniclastic complexes incorporated within Upper Berriasian marls; volcanic lavas and basalt clasts included in the breccias with clay matrix of Upper Oxfordian to Lower Berriasian age, or in brecciated Lower Tothonian calcareous beds of the Early Tithonian. These submarine volcanic activities took place in a carbonate platform environment during the Kimmeridgian to Early Tithonian interval or in a pelagic basin from Late Tithonian onwards. They caused an intense brecciation of Upper Jurassic carbonate formations and a general dismantling of marly calcareous alternations of Upper Tithonian-Lower

Adresse e-mail : benzaggagh@gmail.com. 
Berriasian. Therefore, the Upper Tithonian-Lower Berriasian deposits are marked by frequent stratigraphic gaps in many outcrops of Mesorif, Prerif areas and in the Bou Haddoud nappe.

(c) 2011 Académie des sciences. Published by Elsevier Masson SAS. All rights reserved.

\section{Abridged English Version}

\section{Introduction}

The recent stratigraphic works (Benzaggagh, 2000; Benzaggagh et Habibi, 2006) carried out on the JurassicCretaceous boundary successions of the external Rif chain (Fig. 1) allow us to discover numerous volcaniclastic levels. We present here an inventory of these levels, specifying their importance and their stratigraphic position.

Outcrops of Jurassic-Cretaceous boundary with volcaniclastic levels

\section{Western external Rif area}

In this area, the volcaniclastic levels at the JurassicCretaceous boundary successions are found at numerous locations (Fig. 2).

\section{Outcrops of Jbels Hamama, Mazoura, Kerkor and Alebra}

The stratigraphic succession in these outcrops is formed of a thick silty clay and sandstone alternation from Callovian-Oxfordian age, named "Ferrysch" (Wildi, 1981); a Kimmeridgian-Lower and Upper TithinianUpper Berriasian marly calcereous alternations and marls. Within the carbonate formation (Fig. 4B-D, G), one or several breccias of clay matrix ( 1 to $20 \mathrm{~m}$ thick) are intercalated, formed by calcareous, sandstone clasts and contain basic lavas and basalt clasts (PI. 1. 3, 8, 9). These basic lavas and basalt clasts are also present in cemented calcareous breccia beds of Lower Tithonian age (Pl. 7, 10, 11). On the east side of Jbel Hamama, over the Berriasian marls, a regular volcaniclastic level is located (Fig. 4B), formed by metric blocks of sandstone, limestone (Pl. 6), basalt (Pl. 5) and an interstratified volcanic lava flow (Pl. 4). On the east side of Jbel Ouadine, in Jbel Alebra and in North Mazoura, the marly calcareous alternations are locally absent, but the abundant limestone clasts of Upper Tithonian age, reworked in sedimentary breccias, show that the marly calcareous alternations have been deposited before their general or local post-Tithonian dismantling in relation to the submarine volcanic activities.

\section{Outcrop of oued Zendoula}

In this outcrop, an Upper Jurassic calcareous layer (20 m thick) is exposed showing a sedimentary breccia ( $3 \mathrm{~m})$ on top and volcanic lava (Pl. 12) of latest Early Tithonian age. Other similar volcanic rocks appear in a chaotic zone, $200 \mathrm{~m}$ to the north.
Volcaniclastic complex of douar Harrara and outcrops of North and East Harrara

The first complex is formed by weathered basic lavas included in red marls of the Lower Cretaceous and contains decimetric limestone clasts. This complex is probably synchronous to the volcanic lava flow of the east side of Jbel Hamama. $200 \mathrm{~m}$ to the north, numerous plurimetric volcanic blocks can be found in a chaotic zone containing dismantled beds belonging to the Upper Jurassic formations. $100 \mathrm{~m}$ to the east of douar Harrara, other volcanic rocks appear at the top of the "Ferrysch" formation.

\section{Volcaniclastic complex of douar Kelaa Beni Routène}

The lower part of this complex is formed of a very stratified volcaniclastic layers (Pl. 14) and contains ammonitico rosso lenses, sandstone and limestone clasts from the Upper Jurassic formations. Its higher part is dominated by volcanic lava flows (Pl. 15) covered by Lower Cretaceous marls.

Outcrops of Sidi Kassem, Mguedrouz, Koudiat Bouchta, Chrouf, Kendak El Youdi and Ouldjet El Haffa

In the first outcrop, the top of the "Ferrysch" formation displays some volcanic rocks similar to those exposed north of Harrara and Zendoula. In the east extremity of Jbel Mguedrouz, thin basic lavas, included in ammonitico rosso deposit of Upper Tithonian-Lower Berriasian ag, are found. At Koudiat Bouchta, Upper Berriasian marls contain a level of thin lava flow south of douar Chrouf $(2 \mathrm{~km}$, north of Moulay Bouchta, Fig. 1) and in Kendak El Youdi (4 km, southeast of My Bouchta), Upper Berriasian marls show two or three oxydized levels (1 to $3 \mathrm{~m}$ ) that could correspond to layers of volcanic ashes. At Ouldjet El Haffa ( $5 \mathrm{~km}$, east of My Bouchta), numerous decametric klippe nappes of Upper Jurassic material overthrusted on the Barremian marls. In one of these klippes, the top of the "Ferrysch" formation shows a volcaniclastic level ( 1 to $2 \mathrm{~m}$ thick).

\section{Eastern external Rif area}

In this area, the volcaniclastic levels of the JurassicCretaceous boundary successions have been recognised in Jbel Tahar Bou Zhaier, Jbel Tarhchenna and douar Marticha (Fig. 3).

\section{Jbel Tahar Bou Zhaier}

In this mountain, the Kimmeridgian succession is formed by an alternation of marls and thin limestone 
beds. The Lower Tithonian is formed by a calcareous mass stratified in one to several cemented breccias. Between these two lithostratigraphic units, an important volcanic lava flow with several meters length and a sedimentary breccia of clay matrix ( 1 to $20 \mathrm{~m}$ ) are interstratified (Fig. 4E). Over the calcareous formation, a second breccia with basalt clasts ( 1 to $3 \mathrm{~m}$ ) exists locally. The calcareous beds which surmount it contain calpionellids of the Calpionella alpina zone (Early Berriasian).

\section{Outcrops of douar Marticha and Jbel Tarhchenna}

To the west of douar Marticha, the top of the "Ferrysch » formation shows a regular volcaniclastic level (Fig. 4F). The marly calcareous succession of Kimmeridgian age is marked by a second breccia with basalt clasts. Volcanic materials are also found at the base (Lower Tithonian) and at the top (Upper Lower Tithonian) of the calcareous formation. At the south extremity of Jbel Tarhchenna, the Upper Berriasian marls contain a level of thin lava flow similar to those of Koudiat Bouchta.

Magmatic events, relationship with the sedimentation and palaeogeographic evolution of the external Riffian trough at the Jurassic-Cretaceous boundary

The main periods marked by the submarine volcanic activities are: Latest Oxfordian, Kimmeridgian, Early Tithonian, Early and Late Berriasian. At the OxfordianKimmeridgian boundary, the external Riffian trough had undergone an important palaeogeographic change (Benzaggagh, 2000; Benzaggagh and Atrops, 1997). Its internal area, which corresponded to the deep marine environment during the Oxfordian (Wildi, 1981), formed a middle shallow ridge from Early Kimmeridgian onwards in probable relation with the ascent of the magmatic material. From Late Tithonian onwards, the middle ridge undergoes a rapid collapse accompanied with: submarine volcanic activities; sliding as breccias or as olistoliths; partial or total dismantling of Upper Tithonian marly calcareous alternations and a part of a carbonate platform, as similar to the marls of Lower Berriasian and the top of the "Ferrysch" formation.

\section{Conclusion}

Five episodes of submarine volcanic activities that occur in western as in eastern Rif, have been characterised around the Jurassic-Cretaceous boundary of the external Rif chain. During the Kimmeridgian-Late Berriasian interval (15 Ma), the external Riffian trough displayed two successive palaeogeographic situations: a period of carbonate platform environment during the KimmeridgianEarly Tithonian and a period of basin environment from Late Tithonian onwards. During the first period, the volcanic submarine activities were responsible for the intensive brecciation of the carbonate formation of Mesorif zone and Bou Haddoud nappe. During the second period, these activities caused, in numerous locations, a total or partial dismantling of the Upper Tithonian marly calcareous alternations and the marls of the Lower Berriasian.

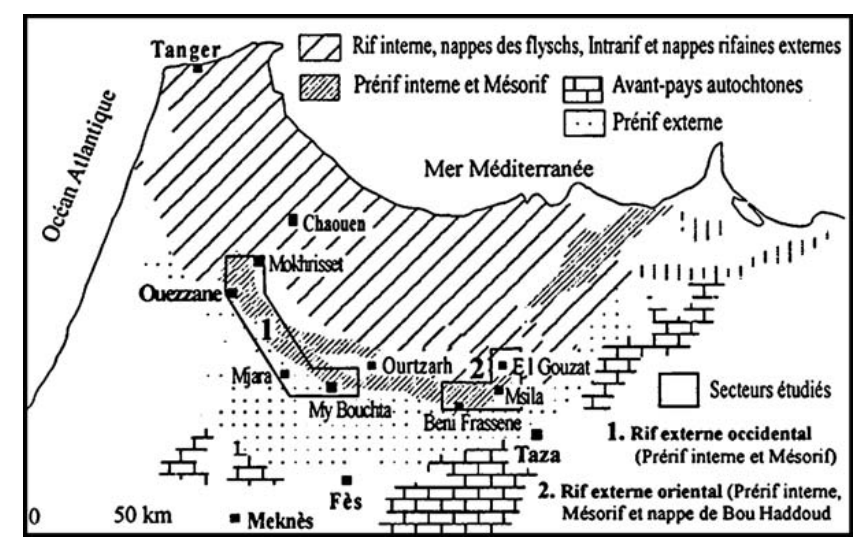

Fig. 1. Carte structurale du Rif (d'après Leblanc, 1979, simplifiée) et localisation des secteurs étudiés.

Fig. 1. Structural map of Rif chain and location of the studied areas.

This dismantling had also affected the whole carbonate platform and the top of the "Ferrysch" formation.

\section{Introduction}

Dans le Rif externe (Fig. 1), il n'est pas rare de rencontrer des masses de roches volcaniques emballées dans des terrains marneux d'âge Méso-Cénozoïque. Ces roches étrangères aux environnements dans lesquels elles sont incorporées, ont été souvent considérées comme des ophites du Trias (Leblanc, 1979 ; Suter, 1964 ; Suter, 1990). Cependant, plusieurs auteurs avaient déjà montré que certains complexes volcanoclastiques du Rif ont un âge plus récent. Ainsi, dans la zone Prédorsalienne des Bokkoya et à la base de la série préflysch de la nappe de Tisirène, des laves basiques ont été datées du Dogger-Malm (Andrieux, 1964 ; Andrieux et Mattauer, 1963). Sur les cartes géologiques à 1/50000 de Beni Frassene et de Dhar Souk, des matériaux volcaniques ont été signalés dans des marnes du Barrémien (Vidal, 1979 ; Vidal, 1983). Un âge Bathonien-Callovien a été attribué à plusieurs complexes volcanoclastiques du Rif externe occidental (Ben Yaich, 1991 ; Ben Yaich et al., 1989). Au Jbel Tifelouest (Intrarif), les calcaires argileux schisteux du Tithonien moyenValanginien inférieur contiennent des lentilles de roches volcaniques interstratifiées (Favre, 1992 ; Vidal, 1983b). Récemment, des analyses géochimiques ont été effectuées (Durand-Delga et al., 2000) sur des échantillons de roches basiques de la zone Prédorsalienne et de la série préflysch de la nappe de Tisirène. Les résultats de ces analyses montrent que ces laves ont un caractère E-MORB, témoignant d'une océanisation au moins partielle du socle du sillon des Flyschs. Les travaux de stratigraphie menés sur les séries du passage Jurassique-Crétacé du Rif externe (Benzaggagh, 2000 ; Benzaggagh et Habibi, 2006) nous ont permis de découvrir plusieurs niveaux marqués par des indices d'activités volcaniques sous-marines. Nous présentons ici un inventaire de ces niveaux, tout en précisant leur importance et leur position stratigraphique. Nous discuterons aussi l'âge attribué à certains complexes volcanoclastiques du Rif externe occidental. Notons que douze échantillons de roches volcaniques de la limite Jurassique-Crétacé du Rif externe, prélevés dans des 


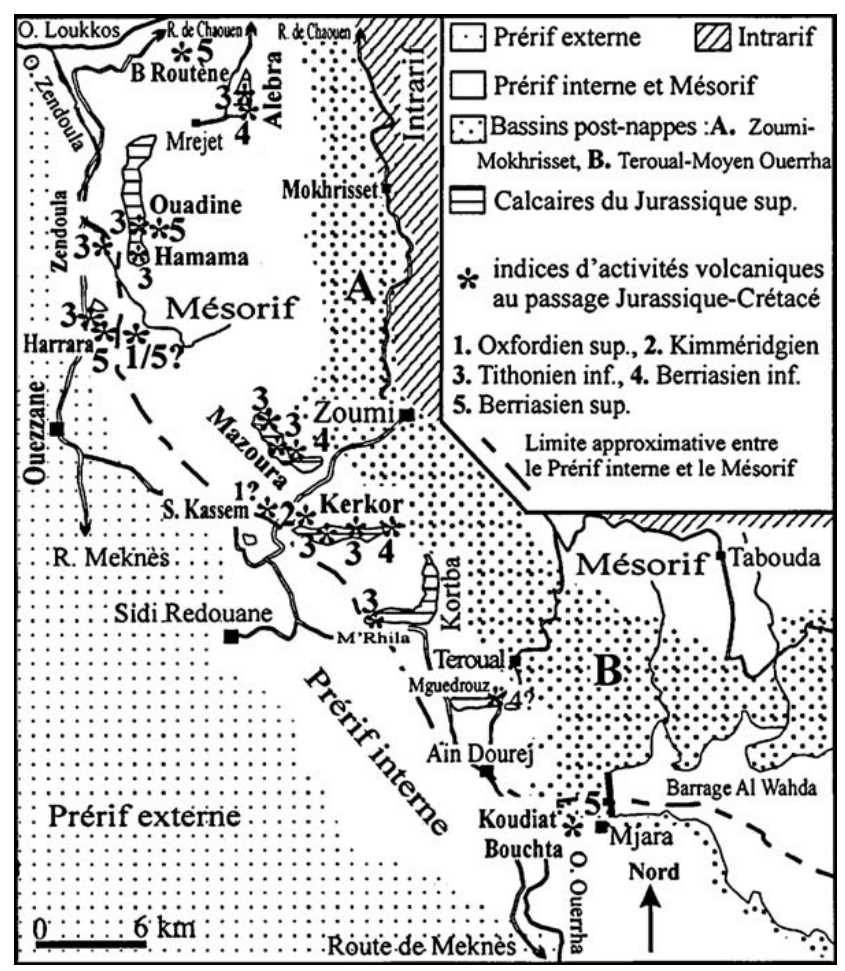

Fig. 2. Carte du Rif externe occidental (d'après Bouhdadi, 1999 ; Suter, $1964,1990)$ et localisation des principaux affleurements montrant des indices d'activités volcaniques sous-marines dans des niveaux de la limite Jurassique-Crétacé.

Fig. 2. Map of western external Rif area and location of the main outcrops with volcaniclastic levels around the Jurassic-Cretaceous boundary.

localités différentes ont été analysés au BRGM (Orléans). Les données préliminaires de ces analyses montrent qu'il s'agit de basalte sous-saturé en silice, ayant un caractère intermédiaire entre MORB normaux et enrichis, témoignant de stades précoces de l'océanisation du socle du sillon rifain externe. L'étude pétrographique et géochimique de ces laves fera l'objet d'un travail en cours de préparation.

\section{Affleurements à niveaux volcanoclastiques dans les séries du passage Jurassique-Crétacé}

Ces affleurements sont plus fréquents dans les séries du Rif externe occidental que dans celles du Rif externe oriental.

\subsection{Rif externe occidental}

Dans cette portion de la chaîne (Fig. 2), des indices d'activités volcaniques sous-marines au passage Jurassique-Crétacé ont été rencontrés dans plusieurs affleurements répartis sur un vaste secteur.

\subsubsection{Jbels Hamama, Mazoura, Kerkor et alebra}

Les séries de ces quatre massifs sont semblables; elles montrent, de bas en haut : le "Ferrysch ", alternance d'argiles et de grès (plus de $1000 \mathrm{~m}$ d'épaisseur) d'âge Callovo-Oxfordien (Wildi, 1981) ; les calcaires du Kimméridgien-Tithonien inférieur (50 à $100 \mathrm{~m}$ ) ; les alternances

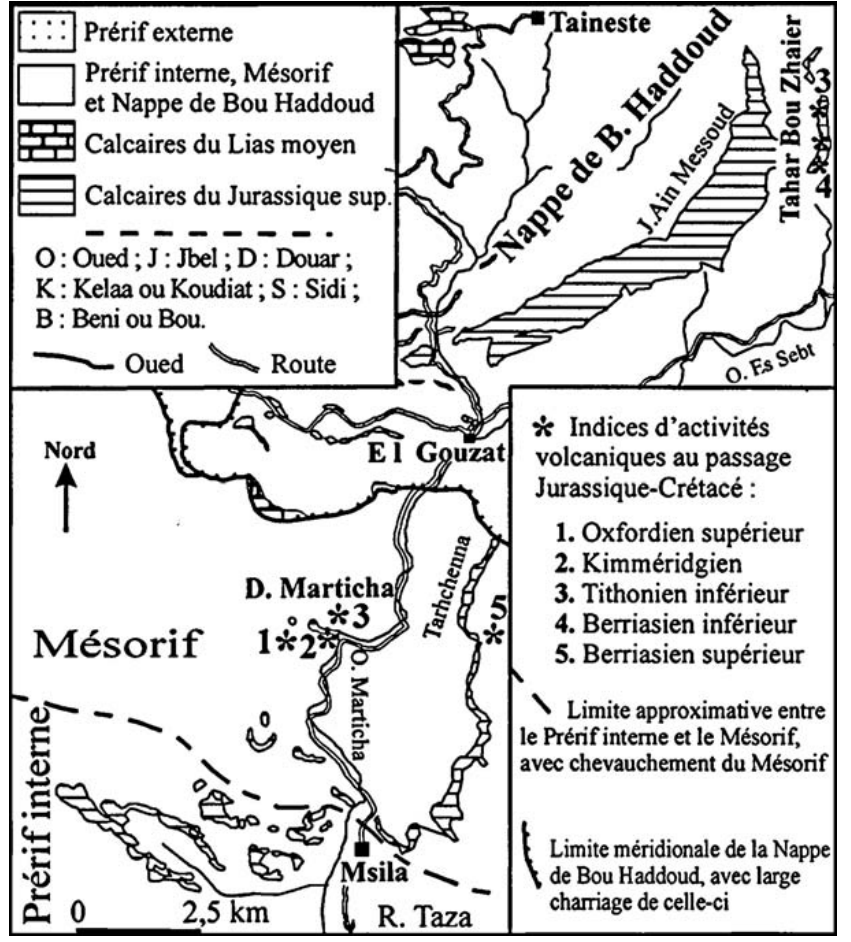

Fig. 3. Carte du Rif externe oriental (d'après Leblanc, 1978, 1983) et localisation des principaux affleurements montrant des indices d'activités volcaniques sous-marines dans des niveaux de la limite JurassiqueCrétacé.

Fig. 3. Map of eastern external Rif area and location of the main outcrops with volcaniclastic levels around the Jurassic-Cretaceous boundary.

marno-calcaires du Tithonien supérieur $(0$ à $50 \mathrm{~m})$ et les marnes du Berriasien (10 à $100 \mathrm{~m}$ ). La formation carbonatée (Fig. 4B-D, G), sous faciès mésorifain (Benzaggagh, 2000 ; Benzaggagh et Habibi, 2006), est formée de successions irrégulières de bancs décimétriques à métriques de calcaires bioclastiques, laminés ( $\mathrm{Pl} .$. 1), bréchiques (Pl. 2) ou microbréchiques. Le microfaciès et la microfaune sont dominés par des formes de plate-forme carbonatée de la zone photique, algues vertes et foraminifères benthiques. Au sein de cette plate-forme, s'intercalent une ou plusieurs brèches à matrice marneuse ( 1 à $20 \mathrm{~m}$ d'épaisseur), constituées d'éléments surtout carbonatés, anguleux et sans grano-classement préférentiel ( $\mathrm{Pl}$. 3), témoignant de glissements sur de faibles distances dans des environnements accidentés. En outre, ces brèches contiennent des éléments gréseux de la formation sous-jacente, des coulées de laves interstratifiées (Pl. 8,9) et des lithoclastes de basalte de forme souvent anguleuse, de taille centimétrique, décimétrique à rarement métrique. Ces lithoclastes sont aussi présents dans des bancs successifs de la formation carbonatée. C'est le cas aux Jbels Alebra (Pl. 10, 11), Kerkor (Pl. 7), Mazoura et Hamama. Dans ces massifs, les brèches, les laves volcaniques et les lithoclastes de basalte sont situés dans des niveaux du Tithonien inférieur (les quatre massifs), du Berriasien inférieur (J. Alebra, milieu de Mazoura) ou du Kimméridgien (J. Kerkor). On note dans ces affleurements une étroite liaison entre la mise en place des brèches et l'apparition du matériel volcanique. 


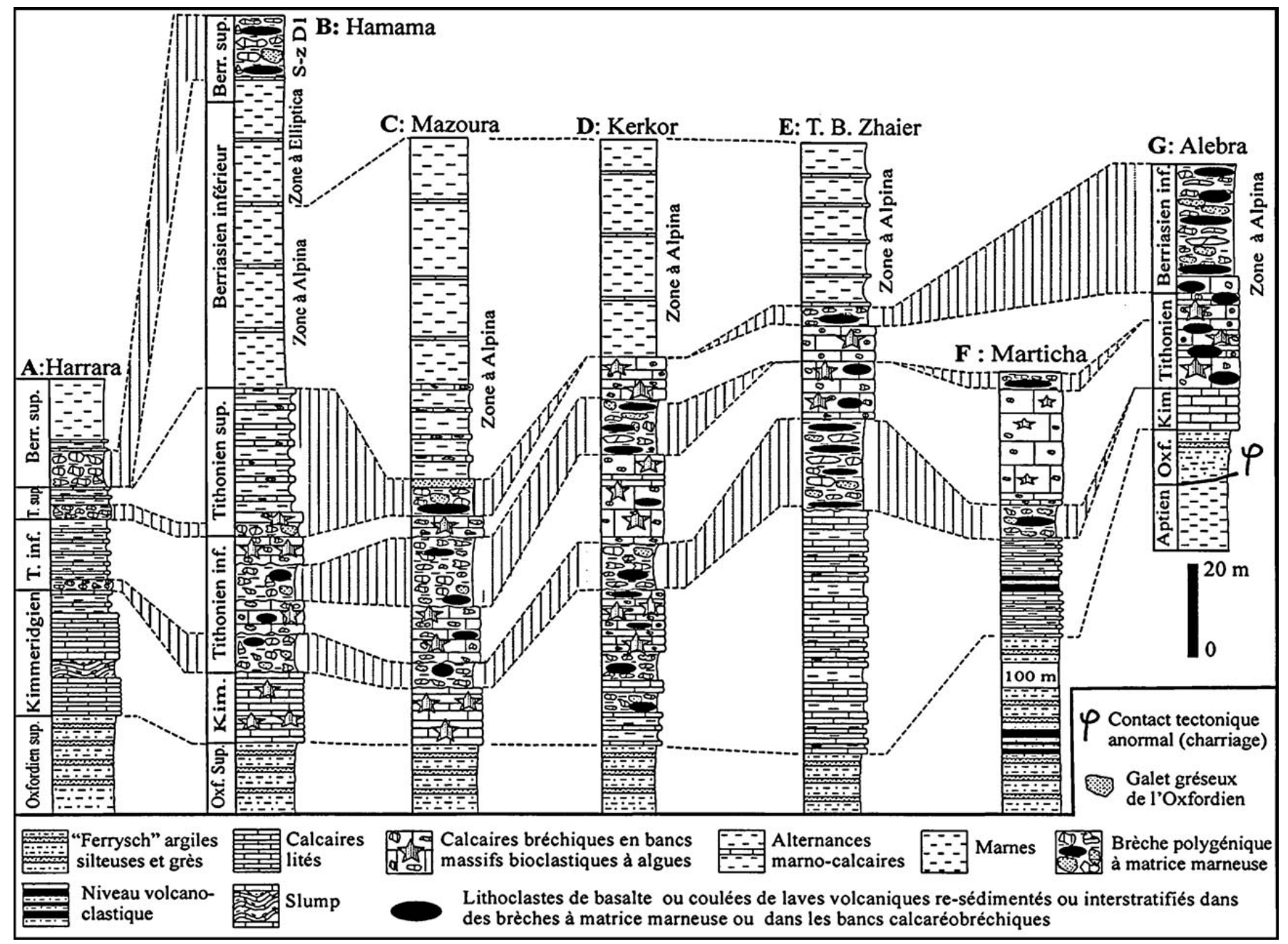

Fig. 4. Logs stratigraphiques des principaux affleurements de la limite Jurassique-Crétacé montrant des indices d'activités volcaniques sous-marines : A, coupe de Harrara nord (Prérif interne occidental) ; B, C, D, G, coupes des Jbels Hamama, Mazoura, Kerkor, Alebra (Mésorif occidental). E, coupe du Jbel Tahar Bou Zhaier (nappe de Bou Haddoud). F, coupe du douar Marticha (Mésorif oriental).

Fig. 4. Stratigraphic logs of the main outcrop sections of the Jurassic-Cretaceous boundary with volcaniclastic levels. A, North Harrara section (western internal Prerif area); B, C, D, G, Hamama, Mazoura, Kerkor and Alebra sections (western Mesorif area); E, Tahar Bou Zhaier section (Bou Haddoud nappe); F, Marticha section (eastern Mesorif area).

Sur le versant est du Jbel Hamama, la série alternante et les marnes sus-jacentes livrent des calpionelles des zones à Chitinoidella boneti et à Crassicollaria du Tithonien supérieur, à Calpionella alpina, C. elliptica et Capionellopsis (sous-zone D1 ; Remane, 1971) du Berriasien. Sur ces marnes repose un niveau volcanoclastique (2 à $3 \mathrm{~m}$ ) qu'on peut suivre sur plusieurs dizaines de mètres (Fig. 4B); il est formé de blocs métriques de grès du "Ferrysch ", de calcaires sous faciès variés, arrachés à la formation carbonatée (Pl. 6), de basalte ( $\mathrm{Pl} .5$ ) et une coulée de lave interstratifiée (Pl. 4). Ce niveau est surmonté par une épaisse masse de "Ferrysch " très chaotique, montrant sur toute son épaisseur des bancs démantelés ou plissés en désordre, témoignant de glissement synsédimentaire en masse hectométrique. Sur le versant est du Jbel Ouadine, au Jbel Alebra et au nord de Mazoura, les alternances du Tithonien supérieur et localement les marnes du Berriasien sont absentes, mais des lithoclastes riches en différentes espèces de Crassicollaria ou de Saccocoma du Tithonien supérieur sont fréquemment rencontrés dans des micro- brèches intercalées au sein des marnes du Berriasien (nord Mazoura, J. Ouadine) ou dans des bancs calcaréobréchiques du sommet de la formation calcaire (J. Alebra). Ceci montre que les alternances du Tithonien supérieur ont été bien déposées avant de subir des démantèlements post-Tithonien, en particulier à la base du Berriasien inférieur et du Berriasien supérieur, en rapport avec les activités volcaniques sous-marines de ces secteurs. Localement, ces démantèlements peuvent affecter toute la plateforme carbonatée et le sommet du "Ferrysch ». C'est le cas à l'extrémité est du Jbel Kerkor, où une lame de " Ferrysch " repose sous forme d'olistolite directement sur les marnes de la base du Berriasien (zone à $C$. alpina) par l'intermédiaire d'une brèche ( 1 à $2 \mathrm{~m}$ d'épaisseur) à éléments variés : gréseux, carbonatés ou volcaniques. Au milieu du Jbel Mazoura, la formation calcaire est surmontée par une épaisse brèche (20 m d'épaisseur) de la base du Berriasien, constituée d'éléments pour l'essentiel carbonatés et contenant de nombreux lithoclastes de basalte. Cette brèche est surmontée par des bancs de calcaires gréseux à 
faciès particulier, puis par une lame allochtone ( 3 à $50 \mathrm{~m}$ d'épaisseur) de grès de la formation " Ferrysch ».

\subsubsection{Affleurements de l'Oued Zendoula}

À l'est du Jbel Bou Rzine, au niveau de l'oued Zendoula, affleure une barre calcaire ( $20 \mathrm{~m}$, ép.), coiffée à son sommet par une brèche sédimentaire ( $3 \mathrm{~m}$, ép.) et une coulée volcanique ( $\mathrm{Pl}$. 12). Les bancs calcaires ont subi des recristallisations thermiques (Pl. 13) qui ont rendu les bioclastes méconnaissables pour des déterminations paléontologiques. Cependant, l'analyse minutieuse de cette coupe montre qu'il s'agit bien de calcaires du Jurassique supérieur présentant une épaisseur, des faciès et des successions lithologiques semblables à ceux des séries mésorifaines de la route de Chaouen (Benzaggagh et Habibi, 2006). Ces calcaires sont donc d'âge KimméridgienTithonien inférieur ; la coulée volcanique principale se place au sommet du Tithonien inférieur. Celle-ci est responsable des recristallisations des bancs calcaires et de la mise en place de la brèche supérieure. À quelques dizaines de mètres au nord, après une lacune de visibilité (sol et végétation), réapparaissent, au sein d'une zone chaotique, de nouveaux blocs de basalte formant la continuité du niveau volcanoclastique ci-dessus.

\subsubsection{Affleurement de Harrara nord}

À $200 \mathrm{~m}$ au nord-ouest du douar Harrara, on rencontre plusieurs filons de roches volcaniques moins altérées et des blocs de basalte, affleurant dans une large zone chaotique constituée d'éléments appartenant aux différentes formations du Jurassique supérieur. Localement, des brèches polygéniques à lithoclastes volcaniques reposent directement sur des bancs métriques de grès du sommet du "Ferrysch ", témoignant d'un démantèlement total de la plate-forme carbonatée. Dans la partie nord de cette zone, des blocs décamétriques de calcaires du Jurassique supérieur, sous faciès prérifain (Benzaggagh, 2000 ; Benzaggagh et Habibi, 2006), affleurent dans un désordre stratigraphique total. Ils sont renversés ou plissés dans une logique non conforme au cadre tectonique postsédimentaire de ce secteur. Il s'agit de blocs ayant subi des glissements sous forme d'olistolites immédiatement après leur dépôt. Toutefois, la coupe la plus complète parmi ces blocs (Fig. 4A) montre trois niveaux de brèches, datées respectivement du Tithonien inférieur, Tithonien supérieur et du Berriasien supérieur (sous-zone D1 des calpionelles). La dernière brèche repose sur des marno-calcaires à Chitinoidella boneti de la base du Tithonien supérieur, ce qui signifie une lacune des zones à Crassicollaria, C. alpina et C. elliptica. Comme dans les cas précédents, ces intervalles lacuneux sont bien représentés par des lithoclastes riches en microfaune du Tithonien supérieur, remaniés dans des brèches ou dans des bancs calcaréobréchiques. Ainsi, le désordre stratigraphique, les brèches et les lacunes partielles ou totales que montrent les séries de ce secteur sont le résultat de glissements synsédimentaires, en particulier au sommet du Tithonien inférieur et à la base du Berriasien supérieur ; glissements que l'on peu mettre en rapport avec des instabilités tectoniques contemporaines de manifesta- tions volcaniques dont les filons de Harrara nord et le complexe du douar Harrara sont les témoins.

\subsubsection{Complexe volcanoclastique du douar Harrara et affleurement de Harrara est}

Le complexe du douar Harrara, plusieurs dizaines de mètres de long et de large, est formé de laves altérées contenues dans une matrice marneuse et renfermant des blocs décimétriques de bancs de calcaires lités, cuits. Ce complexe chaotique est emballé dans des marnes du Crétacé inférieur et il passe à son extrémité nord-est en continuité stratigraphique normale à une formation carbonatée (12 m, ép.) du Jurassique supérieur sous faciès prérifain. La mise en place de ce complexe est probablement Berriasien supérieur, synchrone de la coulée de lave du versant est du Jbel Hamama. Dans le deuxième affleurement, $200 \mathrm{~m}$ à l'est, le sommet du " Ferrysch " contient des roches volcaniques plus ou moins altérées, qui s'apparentent à des filons-couches, dont la mise en place s'intégre dans le cadre des activités volcaniques de ce secteur. Ces filons peuvent avoir un âge fini-Oxfordien à Berriasien supérieur, vu l'absence des formations du sommet du Jurassique et de la base du Crétacé.

\subsubsection{Complexe volcanoclastique de Kelaa des Beni Routène}

Ce complexe de plusieurs dizaines de mètres, moins chaotique que celui du douar Harrara, est situé près de Kelaa des Beni Routène. Sa partie inférieure montre des niveaux volcanoclastiques bien stratifiés (Pl. 14), formés de marnes contenant de minces coulées interstratifiées de laves altérées, des lentilles de marno-calcaires rouges de type ammonitico-rosso et des lithoclastes de grès du " Ferrysch " ou de calcaires du Jurassique supérieur. Sa partie supérieure est dominée par des coulées de laves moins altérées, interstratifiées sous forme de sills (Pl. 15). L'ensemble est coiffé par des marnes du Crétacé inférieur. L'âge de mise en place de ce complexe est certainement Berriasien supérieur.

\subsubsection{Affleurements de Sidi Kassem, Mguedrouz, Koudiat Bouchta, Chrouf, Kendak El Youdi et Ouldjet El Haffa}

Dans le premier affleurement, le sommet du « Ferrysch " est traversé par des filons de roches volcaniques semblables à ceux de Harrara nord et de l'oued Zendoula. Ces filons peuvent avoir un âge sommet Oxfordien à Berriasien. À l'extrémité est du Jbel Mguedrouz, des niveaux ammoniticorosso du Tithonien supérieur-Berriasien inférieur contiennent des laves volcaniques altérées. À Koudiat Bouchta, les marnes du Berriasien supérieur renferment un niveau de minces coulées de laves. Au sud du douar Chrouf ( $2 \mathrm{~km}$ au nord de Moulay Bouchta, Fig. 1) et à Kendak El Youdi ( 4 km au sud-est de My Bouchta), les marnes du Berriasien supérieur (zone à Calpionellopsis) montrent deux à trois niveaux ( 1 à $3 \mathrm{~m}$, d'épaisseur) rouges à oxydes de fer, pouvant correspondre à des dépôts marneux à cendres volcaniques. À Ouldjet El Haffa (5 km à l'est de My Bouchta), plusieurs klippes de nappes à matériel Jurassique supérieur (" Ferrysch » et calcaires mésorifains) reposent en contact plat sur les marnes du Barrémien. Dans l'une de ces klippes, le sommet du " Ferrysch ", immédiatement sous la masse calcaire, montre un niveau volcanoclastique interstratifié de 1 à $2 \mathrm{~m}$ d'épaisseur. 

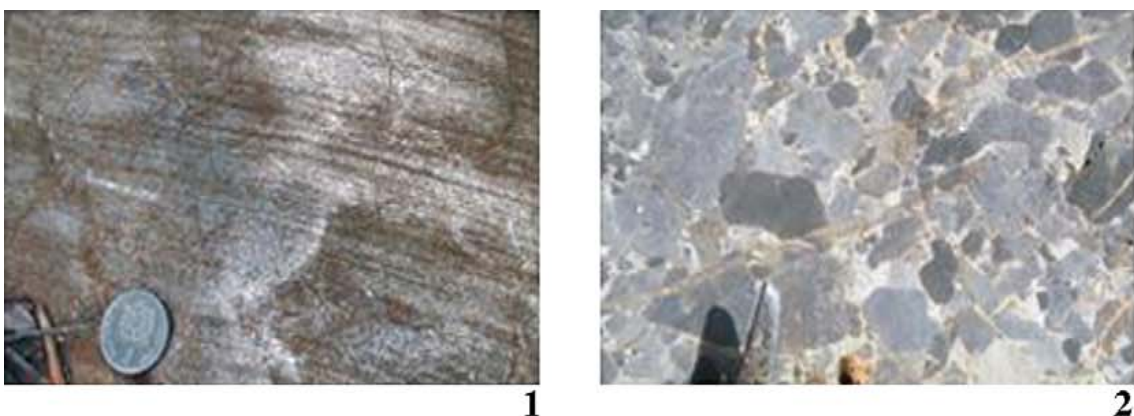

1
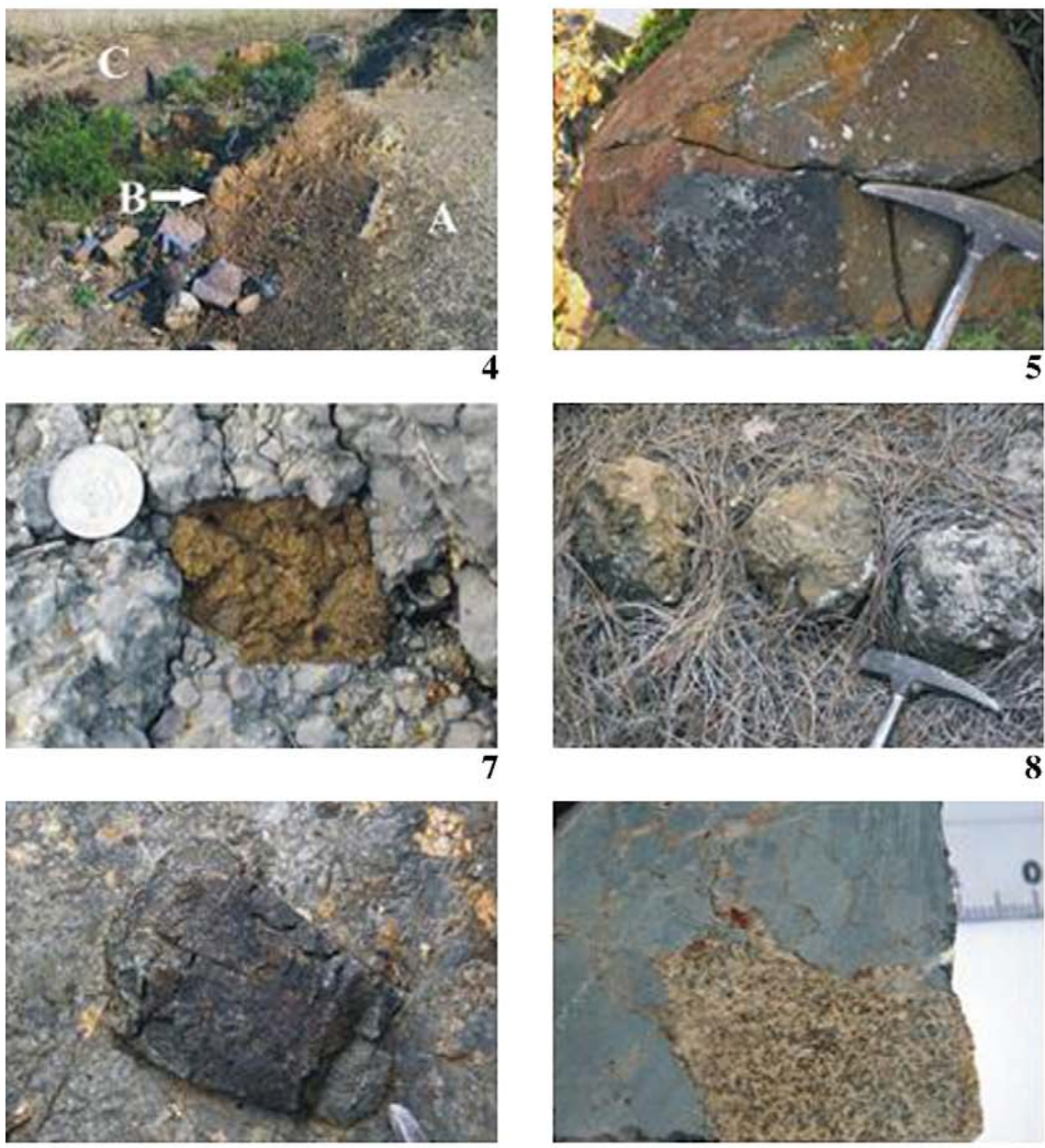

11

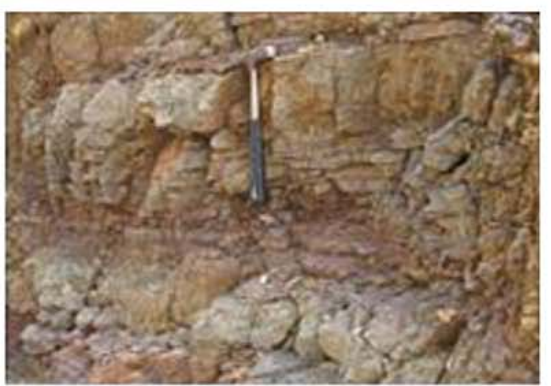

14
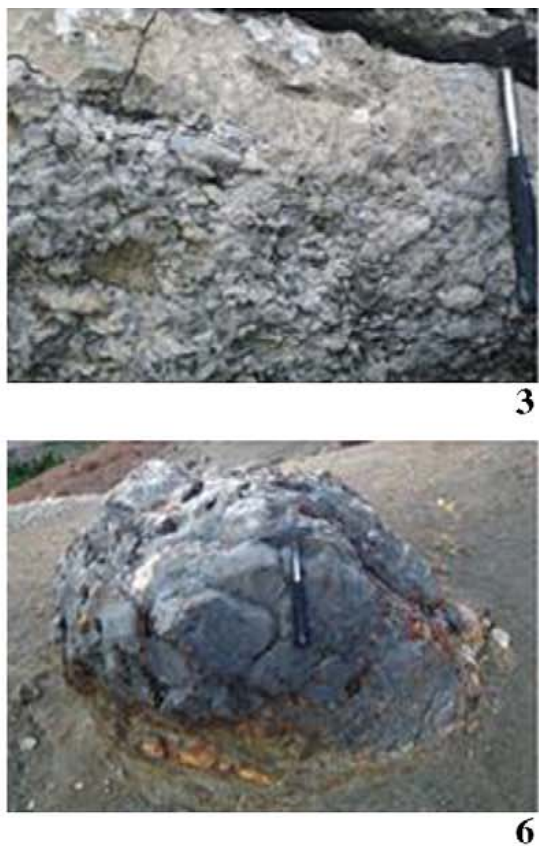

6
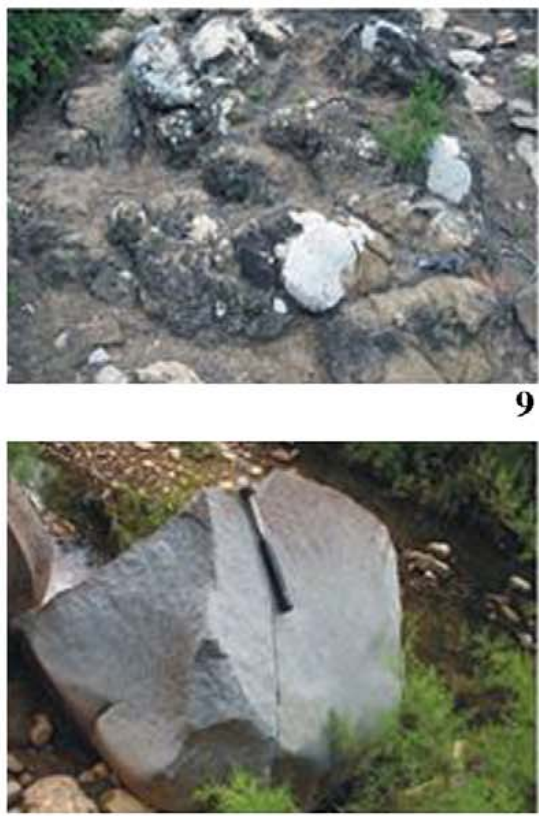

12

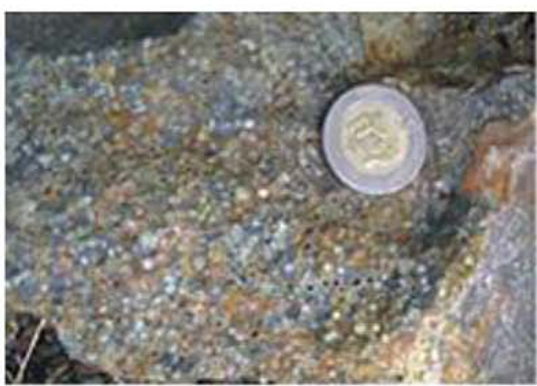

15

Planche 1. 1. Banc de calcaire laminé ; Tithonien inférieur, Jbel Alebra. 2. Brèche cimentée à éléments carbonatés anguleux provenant du démantèlement sur place des bancs sous-jacents ; Tithonien inférieur, J. Mazoura. 3. Brèche semi-cimentée à éléments anguleux, essentiellement carbonatés, contenant un lithoclaste volcanique et passant vers le haut à un banc micro-bréchique ; Tithonien inférieur, J. Mazoura. 1 à 3 . Faciès caractéristiques des séries carbonatées du Mésorif. 4. Coulée volcanique interstratifiée (B) reposant sur les marnes du Berriasien supérieur (A), surmontée par une zone chaotique renfermant des blocs métriques de grès, de calcaires et de basalte $(\mathbf{C})$; versant est du J. Hamama. $\mathbf{5}$. Bloc de basalte dans la zone chaotique ; $100 \mathrm{~m}$ au sud de la localité précédente. 6. Bloc de calcaire du Jurassique supérieur emballé sous forme d'olistolite dans les marnes du Berriasien supérieur, immédiatement sous la zone chaotique. Noter la forme arrondie et l'aspect émoussé du bloc calcaire. $\mathbf{7}$ et 10. Lithoclastes de roches volcaniques, resédimentés dans des 


\subsection{Rif externe oriental}

Dans ce secteur (Fig. 3), des témoins d'activités volcaniques sous-marines à la limite Jurassique-Crétacé ont été rencontrés aux Jbels Tahar Bou Zhaier, Tarhchenna et au douar Marticha.

\subsubsection{Jbel Tahar Bou Zhaier}

Il appartient à l'extrémité sud de la nappe de Bou Haddoud (Leblanc, 1978 ; Leblanc, 1979). Les calcaires du Jurassique supérieur (Fig. 4E) y ont une épaisseur variable (30 à $100 \mathrm{~m}$ ) et un pendage est. La série du Kimméridgien (10 à $50 \mathrm{~m}$ ) est constituée de calcaires en bancs lités. Le Tithonien inférieur ( 5 à $30 \mathrm{~m}$ ) forme une masse compacte organisée en une ou plusieurs brèches bien cimentées à éléments carbonatés et contenant des lithoclastes de grès ou de basalte. Entre ces deux unités lithostratigraphiques s'intercale une épaisse brèche à matrice marneuse ( 1 à $20 \mathrm{~m}$, d'épaisseur) datée de la base du Tithonien. Cette brèche est constituée d'éléments carbonatés, gréseux ou volcaniques de taille parfois métrique, passant latéralement à une coulée de lave interstratifiée sous forme de sill, sur plusieurs dizaines de mètres de long. La présence de fréquents lithoclastes de basalte dans des niveaux successifs de la formation calcaréo-bréchique du Tithonien inférieur et la bréchification intense de celle-ci, montrent que, dans ce secteur, les activités volcaniques se sont poursuivies tout au long du Tithonien inférieur. Au-dessus de la masse calcaire repose localement une seconde brèche ( 1 à 3 m d'épaisseur), à éléments volcaniques. Les niveaux marno-calcaires qui la surmontent fournissent des calpionelles de la zone à $C$. alpina. Les alternances du Tithonien supérieur ont subi des démantèlements post-dépôt en rapport avec des activités volcaniques, comme il est le cas dans la plupart des affleurements du Rif occidental.

\subsubsection{Affleurements du douar Marticha et du Jbel Tarhchenna}

Aux environs du douar Marticha affleurent plusieurs petits blocs calcaires (10 à $20 \mathrm{~m}$, ép.) nommés "Sofs ", reposant en continuité stratigraphique normale sur le "Ferrysch ». À $120 \mathrm{~m}$ sous la base de ces blocs, le sommet du "Ferrysch " montre un niveau volcanoclastique interstratifié constitué d'argiles silteuses contenant des lithoclastes de grès ou de basalte et des minéralisations d'oxydes de fer (Fig. 4F). Ce niveau se poursuit latéralement sur plusieurs dizaines de mètres au sein du "Ferrysch ». À $20 \mathrm{~m}$ sous les blocs calcaires, on rencontre dans des marno-calcaires du Kimméridgien, une seconde brèche interstratifiée ( 1 à $3 \mathrm{~m}$ ) à éléments gréseux, marnocalcaires et volcaniques. Au pied des escarpements calcaires, dans des niveaux de la base du Tithonien, se développe une troisième brèche à éléments variés, en particulier volcaniques. Sur la surface supérieure de certains blocs (sommet du Tithonien inférieur) reposent de minces coulées de lave. À l'extrémité sud du Jbel Tarhchenna, les marnes du Berriasien supérieur (zone à Calpionellopsis) contiennent de minces coulées de laves semblables à celles de Koudiat Bouchta.

\section{Discussion sur l'âge Bathonien-Callovien attribué à des complexes volcanoclastiques du Rif externe occidental}

On a attribué (Ben Yaich, 1991 ; Ben Yaich et al., 1989) un âge Bathonien-Callovien à la mise en place des principaux complexes volcanoclastiques du Rif externe occidental, en particulier ceux de Harrara et de Beni Routène. Les arguments avancés par ces auteurs sont : la présence dans le complexe de Harrara de lithoclastes de calcaires à filaments, considérés comme d'âge Bathonien ; la prédominance, dans ces deux complexes, de lentilles de marno-calcaires rouges supposées issues du démantèlement de l'ammonitico-rosso du Bajocien et l'absence de lithoclastes de grès, prise comme argument pour une mise en place antérieure au dépôt du « Ferrysch ». Ces arguments ne sont malheureusement pas déterminants car, d'une part, dans les séries du Rif externe, les filaments sont souvent abondants dans les niveaux des zones à Crussolliceras divisum et Hybonoticeras Beckeri du Kimméridgien (Benzaggagh, 2000). Les faciès Bathonien à filaments du Rif externe se présentent sous forme de plaquettes minces, feuilletées et microplissées à aspect de schiste (affleurement de Mjara). Ce type de plaquettes n'est pas représenté dans ces deux complexes. D'autre part, des faciès rouges de type ammonitico-rosso sont bien représentés dans les séries du Jurassique supérieur du Prérif interne et du Mésorif, entre autres : l'ammonitico-rosso marno-calcaire de la zone à Microcanthum du versant est du Jbel Hamama et de l'oued Zendoula, à l'embranchement de la piste du

bancs calcaréo-bréchiques bien cimentés ; Tithonien inférieur (7. J. Kerkor, 10. J. Alebra). 8. et 9. Basalte en boules sphériques et importante coulée de lave, contenus dans la brèche du sommet du Tithonien inférieur ; extrémité est du J. Kerkor. 11. Échantillon scié dans un banc calcaréo-bréchique du Tithonien inf. du J. Alebra, renfermant un lithoclaste volcanique. L'aspect du contact entre le lithoclaste et la matrice carbonatée montre que la mise en place du lithoclaste volcanique est synsédimentaire, antérieure à la diagenèse du banc calcaire. 12 et 13. Bloc de basalte moins altéré et banc de calcaire recristallisé sous l'effet de la lave volcanique ; Tithonien inférieur, coupe de l'oued Zendoula. 14. et 15. Niveaux volcano-sédimentaires bien stratifiés et lave volcanique montrant des cavités de dégazage ; parties inférieure (14) et supérieure (15) du complexe des Beni Routène, Berriasien supérieur.

Planche 1. 1. Laminated calcareous bed; Lower Tithonian, Jbel Alebra. 2. Cemented breccia with angular carbonate clasts derived from in situ dismantling of the underlying strata; Lower Tithonian, J. Mazoura. 3. Semi-cemented breccia with angular carbonate clasts and containing a volcanic clast. Its upper part forms a microbrecciated calcareous bed; Lower Tithonian, J. Mazoura. 1 to 3. Characteristic deposits of Late Jurassic mesoriffian carbonate formation. 4. Interstratified volcanic lava (B) overlying the Upper Berriasian marls (A) and underlying a chaotic volcaniclastic level (C) containing metric blocks of sandstone, limestone and basalt; east side of J. Hamama. 5. Basalt block within the volcaniclastic level; $100 \mathrm{~m}$ south of the previous locations. 6 . Late Jurassic calcareous block reworked as olistolith in the Upper Berriasian marls, situated immediately under the volcaniclastic level. Note rounded edges of the calcareous block. 7 and 10. Basalt clasts included in cemented calcareous brecciated beds; Lower Tithonian (7. J. Kerkor, 10. J. Alebra). 8 and 9. Basalt lava with spheroidal forms and an important volcanic lava level contained in the late Lower Tithonian breccia; east extremity of J. Kerkor. 11. Sample sawed in brecciated calcareous bed of Early Tithonian strata from J. Alebra containing a basalt clast. The contact between the volcanic clast and the carbonate matrix shows that the setting up of the volcanic clast is synchronous to sedimentation, prior to diagenesis of the calcareous bed. 12 and $\mathbf{1 3}$. Less weathered basalt block and calcareous bed recrystallized by the effect of the volcanic lava; Lower Tithonian, oued Zendoula section. 14 and $\mathbf{1 5}$. Stratified volcaniclastic levels and basaltic lava showing degassing cavities; lower (14) and higher part (15) of Beni Routene volcaniclastic complex, Upper Berriasian. 
douar El Ouadine. Enfin, l'absence ou la rareté des lithoclastes de grès dans le complexe de Harrara ne signifie pas obligatoirement une mise en place antérieure au dépôt du " Ferrysch ", car dans la plupart des cas, les glissements intéressent plus particulièrement la pellicule superficielle de la pile lithostratigraphique. On peut aussi signaler que les complexes de Harrara et des Beni Routène sont emballés dans des marnes du Crétacé inférieur et ils ne montrent aucun contact stratigraphique avec les séries du Lias ou du Dogger anté-« Ferrysch » qui n'affleurent nulle part dans cette partie de la chaîne.

\section{Succession des événements magmatiques, relation volcanisme-sédimentation et évolution paléogéographique du sillon rifain externe à la limite Jurassique-Crétacé}

Le sillon rifain externe, en particulier sa partie médiane, a été le siège de plusieurs manifestations volcaniques sousmarines entre le sommet de l'Oxfordien et la base du Berriasien supérieur, dont les principales ont eu lieu : 1- au sommet de l'Oxfordien et $\mathbf{2}$ - pendant le Kimméridgien, avec des activités restreintes, limitées aux secteurs de Marticha, Kerkor, Ouldjet El Hafa et probablement à Sidi Kassem et Harrara est ? ; 3- au Tithonien inférieur, avec des manifestations importantes qui se sont exprimées dès la base de cet étage à Tahar Bou Zhaier, Marticha et probablement à Mazoura, Hamama, Alebra et Kerkor. Ces manifestations se sont poursuivies tout au long du Tithonien inférieur à Tahar Bou Zhaier, Hamama, Mazoura, Kerkor, Alebra, Harrara nord, Marticha et Zendoula ; 4.- au Berriasien inférieur et 5- au Berriasien supérieur, avec des manifestations importantes qui ont été enregistrées dans les secteurs d'Alebra, Kerkor, Mazoura, Hamama, Mguedrouz, Harrara, Beni Routène, Tahar Bou Zhaier, Koudait Bouchta et Tarhchenna.

Au passage Oxfordien-Kimméridgien le sillon rifain externe a subi un important bouleversement paléogéographique (Benzaggagh et Atrops, 1997). Ses parties internes, en particulier le Mésorif et localement l'Intrarif, qui correspondaient à des milieux profonds pendant l'Oxfordien (Wildi, 1981), formaient dès le Kimméridgien une ride médiane très peu profonde. Il est probable que la remontée du matériel magmatique chaud à partir de l'Oxfordien supérieur à travers la zone mésorifaine a entraîné un bombement de cette zone. Au Tithonien supérieur-Berriasien, la ride mésorifaine subira un effondrement rapide, mais saccadé, en particulier à la base du Tithonien supérieur, du Berriasien inférieur et du Berriasien supérieur. Ces affaissements ont été accompagnés par des phénomènes particuliers : épanchements volcaniques sous-marins ; glissements sous forme de brèches ou d'olistolites ; démantèlement local, total ou partiel, des alternances du Tithonien supérieur, voire de toute la plate-forme carbonatée et intenses remaniements dans des niveaux du Berriasien. Ainsi, dans la plupart des affleurements du Rif occidental, les niveaux du Tithonien supérieur et en partie ceux du Berriasien inférieur sont absents. Ces niveaux ont été démantelés immédiatement après leur dépôt. C'est le cas aux Jbels Alebra, Mazoura, Kerkor, Ouadine, Mrhila, Kortba, Mguedrouz, Harrara et dans les affleurements situés de part et d'autre de l'oued Zendoula, au nord du Jbel Bou Rzine.
Dans le Rif externe oriental, les niveaux du Tithonien pro parte et de la base du Berriasien sont absents dans plusieurs coupes des secteurs de Beni Frassene, Lahnassar sud (à l'ouest de Msila) et au Jbel Tahar Bou Zhaier.

\section{Conclusion}

Les séries de la limite Jurassique-Crétacé du Rif externe enregistrent cinq épisodes d'activités volcaniques sousmarines qui se rencontrent, aussi bien dans les séries des secteurs occidentaux, que dans celles des secteurs orientaux de la chaîne. La présence de faune, surtout de calpionelles, a permis d'attribuer des âges précis à la plupart de ces manifestations. On distingue ainsi des activités volcaniques sous-marines au sommet de l'Oxfordien, au Kimméridgien, mais surtout pendant le Tithonien inférieur, à la base du Berriasien inférieur et du Berriasien supérieur. Entre le sommet de l'Oxfordien et le Berriasien (environ $15 \mathrm{Ma}$ ), le sillon rifain externe a connu deux situations paléogéographiques successives : une période de plate-forme carbonatée au Kimméridgien-Tithonien inférieur et une période de bassin à partir du Tithonien supérieur. Pendant la première période, les activités volcaniques ont été responsables de la bréchification intense des séries carbonatées du Mésorif et de la nappe de Bou Haddoud. Au cours de la seconde période, ces manifestations ont entraîné le démantèlement total, dans plusieurs secteurs, des alternances du Tithonien supérieur et localement des marnes du Berriasien, voire parfois de toute la plate-forme carbonatée du sommet du Jurassique et d'une partie de la formation " Ferrysch ». Il est probable que les matériaux volcaniques des nappes des Flyschs et du Jbel Tifelouest (Intrarif) sont synchrones des roches volcaniques du Mesorif et de la nappe de Bou Haddoud. Ainsi, la répartition géographique large de ce matériel volcanique au sein des séries de la limite JurassiqueCrétacé révèle une océanisation précoce et générale du socle du sillon rifain s.l. (Rif externe et sillon des Flyschs). Il semble que cette tendance à l'océanisation a été vite avortée à partir du début du Crétacé supérieur par la rotation anti-horaire de l'Ibérie et la dérive de l'Afrique vers le nord-est puis vers le nord pendant le Tertiaire, entraînée par l'ouverture de l'Atlantique sud.

\section{Remerciements}

Ce travail a été réalisé dans le cadre du projet PROTARS III $n^{\circ}$ D 15/07 (Université Moulay Ismail, Meknès). L'auteur tient à remercier vivement le Professeur Michel DurandDelga pour la révision de la version française du manuscrit et pour ses précieuses remarques et suggestions scientifiques ; ainsi que le Professeur Philips Rossi pour l'analyse au BRGM à Orléans de douze échantillons de roches volcaniques du Rif externe.

\section{Références}

Andrieux, J., 1964. Présence de roches vertes d'âge Secondaire dans les Bokoya (Rif, Maroc). C. R. somm. Soc. geol. France 7, 252-253.

Andrieux, J., Mattauer, M., 1963. Sur la présence de roches vertes à la base des nappes " ultra " du Rif. C. R. somm. Soc. geol. France 7, 213-215. 
Ben Yaich, A., 1991. Évolution tectono-sédimentaire du Rif externe centro-occidental (régions de Msila et d'Ouezzane, Maroc). La marge africaine du Jurassique au Crétacé. Les bassins néogènes d'avantfosse. Thèse d'Etat, Univ. Pau, 308 p.

Ben Yaich, A., Hervoüet, Y., Duée, G., El Hatimi, N., 1989. Âge jurassique et réinterprétation des roches basiques au Nord de Ouezzane (Rif externe, Maroc) ; signification géodynamique. C. R. Acad. Sci. Paris, Ser. II 309, 1197-1202.

Benzaggagh, M., 2000. Le Malm supérieur et le Berriasien dans le Prérif interne et le Mésorif (Rif, Maroc). Biostratigraphie, lithostratigraphie, paléogéographie et évolution tectono-sédimentaire. Docum. Lab. Géol. Lyon 152, 247.

Benzaggagh, M., Atrops, F., 1997. Le Malm supérieur et le Berriasien dans le Prérif interne et le Mésorif (Rif, Maroc) : stratigraphie et paléogéographie. Eclogae Geol. Helv. 90, 513-529.

Benzaggagh, M., Habibi, M., 2006. Les séries carbonatées du Jurassique supérieur et les niveaux de passage au Crétacé inférieur dans la partie occidentale du Rif externe (Prérif interne et Mésorif, Maroc). Stratigraphie, paléogéographie et évolution tectonosédimentaire. Newsl. on Stratigr. 42 (2), 115-141.

Bouhdadi, S., 1999. Carte géologique du Rif, Zoumi à 1/50000. Notes Mem. Serv. Geol. Maroc 386.

Durand-Delga, M., Rossi, P., Olivier, P., Puglisi, D., 2000. Situation structurale et nature ophiolitique de roches basiques jurassiques associées aux flyschs maghrébins du Rif (Maroc) et de Sicile (Italie). C. R. Acad. Sci. Paris, Ser. II a 331, 29-36.
Favre, P., 1992. Géologie des massifs calcaires situés au front sud de l'unité de Ketama (Rif, Maroc). Publ. Dépt. Géol. Paléont. Univ. Genève 11, 138.

Leblanc, D., 1978. Carte géologique du Rif, Bab El Mrouj-Taza Nord à 1/ 50000. Notes Mem. Serv. Geol. Maroc 287.

Leblanc, D., 1979. Étude géologique du Rif externe oriental au nord de Taza (Maroc). Notes Mem. Serv. Geol. Maroc 281, 159.

Leblanc, D., 1983. Carte géologique du Rif, Taineste à 1/50000. Notes Mem. Serv. Geol. Maroc 305 .

Remane, J., 1971. Les Calpionelles, Protozoaires planctoniques des mers mésogéenes de l'époque secondaire. Extr. Ann. Guéb. $47^{\mathrm{e}}$ année, pp. $1-25$.

Suter, G., 1964. Carte géologique du Rif, région du Moyen Ouerrha, Tafrannt de l'Ouerrha-Moulay Bou Chta à 1/50000. Notes Mem. Serv. Geol. Maroc 165 .

Suter, G., 1990. Carte géologique du Rif, Terwal-Oulad Aïssa à 1/50000. Notes Mem. Serv. Geol. Maroc 224

Vidal, J.C., 1979. Carte géologique du Rif, Beni Frassene à 1/50000. Notes Mem. Serv. Geol. Maroc 283.

Vidal, J.C., 1983. Carte géologique du Rif, Dhar Souk à 1/50000. Notes Mem. Serv. Geol. Maroc 298 .

Vidal, J.C., 1983b. Notice explicative de la carte géologique du Rif, Dhar Souk à 1/50000. Notes Mem. Serv. Geol. Maroc 298 .

Wildi, W., 1981. Le Ferrysch : cône de sédimentation en eau profonde de la bordure nord-ouest de l'Afrique au Jurassique moyen et supérieur (Rif externe, Maroc). Eclogae Geol. Helv. 74, 481-527. 